\title{
Possible role of helper and cytolytic $T$ cells in mycobacterial infections
}

\author{
S H E KAUFMANN, S CHIPLUNKAR, I FLESCH \& G DE LIBERO \\ Max-Planck-Institut für Immunbiologie, Stübeweg 51, 78 Freiburg, \\ FRG
}

\section{Introduction}

During the past years it has become increasingly clear that distinct and stable types of peripheral $\mathrm{T}$ cells can be defined by virtue of their cell surface phenotype and the way how they recognize antigen: $\mathrm{CD} 4 \mathrm{~T}$ cells $\left(\mathrm{L} 3 \mathrm{~T} 44^{+}\right.$in mice, $\mathrm{T}^{+}+$in man) see antigen in the context of class II molecules and CD8 $\mathrm{T}$ cells (Lyt2+ in mice, $\mathrm{T}^{+}+$in man) recognize antigen in association with class I molecules (1).

Initially, it was thought that this phenotypic and genetic dichotomy also reflected a strict functional dissociation, with CD4 $\mathrm{T}$ cells being responsible for the delivery of helper/inducer signals and CD8 $\mathrm{T}$ cells for target cell lysis. Although we now know that this funcitional dissociation is less stringent it still appears that the two T-cell sets have a predilection for either function.

Why is this important for a better understanding of leprosy and tuberculosis? It has been proposed that the T-cell dichotomy had evolved as a strategy for optimal host defence against intracellular infections $(2,3)$. Viruses can infect a variety of host cells and their replication strictly depends on the viability of the latter. Hence, target cell lysis should be an efficient eradication mechanism for viral infections. Furthermore, virtually all nucleated cells express class I molecules ensuring detection of any virus-infected cell by class I-restricted T lymphocytes. In contrast, intracellular bacteria are primarily localized in mononuclear phagocytes, but can also multiply in the extracellular space. Macrophages possess a variety of inducible antimicrobial capacities. Hence, macrophage activation should be highly effective in controlling intracellular bacterial infections. Furthermore and unlike most other cells, mononuclear phagocytes express class II molecules affording selective recognition by helper/inducer T lymphocytes.

A t first sight, one might consider the second mechanism as being fully sufficient for host defence against tuberculosis and leprosy. At second sight, however, conditions can be envisaged where macrophage activation may not be sufficient. In principle there are two major obstacles against effective elimination of mycobacteria by helper/inducer T-cell mediated mechanisms alone.

(1) Mycobacterium tuberculosis and M. leprae, the etiologic agents of tuberculosis and leprosy, respectively, have developed highly effective evasion mechanisms for survival in macrophages. These include: resistance to reactive oxygen metabolites and lysosomal enzymes, inhibition of phagosome-lysosome fusion, and/or evasion into the cytoplasm (4).

(2) Mononuclear phagocytes are highly heterogeneous in their antimicrobial potential and in tissue macrophages much weaker antibibacterial capacities can be activated than in blood monocytes (5). Furthermore, mycobacteria can enter certain nonprofessional phagocy- 
tes; e.g., in tuberculoid leprosy, M. leprae preferentially inhabits Schwann cells (6). Thus, mycobacteria which are capable of entering host cells expressing antimicrobial functions against which they are resistant will find niches which allow or even afford their survival. In addition, inside such cells, mycobacteria can drastically reduce their metabolic activity and become transiently resistant to antibiotics.

It is easily conceivable that in such instances, lysis of infected host cells can represent a beneficial adjunct to helper/inducer T-cell mediated mechanisms. In the following, experiments will be summarized which show that helper and cytolytic T lymphocytes become demonstrable after immunization with $M$. tuberculosis and $M$. leprae and the possible contribution of both T-cell activities to host defence will be dicussed.

\section{Results}

We have generated T-cell clones from mice immunized with M. leprae or M. tuberculosis and used these clones as tools for analysis of the relative contribution of helper and cytolytic $\mathrm{T}$ cells to the immune response in leprosy and tuberculosis (7-11).

\section{MYCOBACTERIA REACTIVE CD8 T-CELL CLONES}

Mice were immunized intradermally with 107 irradiated $M$. leprae organisms (kindly provided by Dr. R.J.W. Rees) and after 5 weeks, draining lymph-node cells were harvested (10). Lymph node cells $\left(2 \times 10^{5} / \mathrm{ml}\right)$ were cultured in the presence of $1 \mu \mathrm{g} / \mathrm{ml}$ soluble $M$. leprae proteins (kindly provided by Dr. R.J.W. Rees), and $2 \times 105 / \mathrm{ml}$ irradiated (3300R) spleen cells as accessory cells (AC). After 1 week of culture, cells were restimulated with AC, antigen and $10 \%$ interleukin 2 (IL-2) containing supernatant from Concanavalin A activated rat spleen cells (ConA-SN). These cells were expanded and propagated in vitro by alternate cycles of restimulation with ConA-SN alone or with antigen, AC, and ConA-SN. A cell line was cloned under limiting dilution conditions in the presence of $\mathrm{AC}$, antigen, and ConA-SN and cloned $\mathrm{T}$ cells were grown up as described above.

Alternatively, mice were immunized subcutaneously with $2 \mathrm{mg}$ killed, lyophilized $M$. leprae (kindly provided by Dr. P.J. Brennan) in incomplete Freund's adjuvant and after 8 days, draining lymph node cells were collected. Cells were restimulated in vitro with $10 \mu \mathrm{g} /$ $\mathrm{ml} M$. leprae, $2 \times 105 / \mathrm{ml} \mathrm{AC}$ and $101 \mathrm{ConA}-\mathrm{SN}$ and grown up as described above.

$M$. tuberculosis reactive $\mathrm{T}$-cell clones were estabished from lymph node cells of mice immunized subcutaneously with killed lyophilized $M$. tuberculosis H37Ra (purchased from Difco) in incomplete Freund's adjuvant (11). Eight days after immunization, cells were harvested and restimulated with $2 \times 10^{5} / \mathrm{ml} \mathrm{AC}, 25 \mu \mathrm{g} / \mathrm{ml} \mathrm{M}$. tuberculosis, and $10 \%$ ConA-SN. After 8 days, cells were cloned under limiting dilution conditions in the presence of AC, antigen, and ConA-SN and expanded as described. T-cell clones from M. leprae or M. tuberculosis immune mice established in this way had the phenotype Thyl+, L3T4 $\div$, Lyt $2+$ and hence belonged to the CD8 T-cell set.

For determination of the cytolytic activities of these T-cell clones towards host cells expressing mycobacterial antigens, bone marrow macrophages (BMM $\varnothing$ ) were used as target cells. Bone marrow cells were cultured in hydrophobic teflon bags in medium supplemented with conditioned medium from L929 cells for 9 to 14 days as described $(12,13)$. Graded numbers of purified $\mathrm{T}$ cells were added to $10^{5} \mathrm{BMM} \varnothing$ in the presence or absence of appropriate antigen in flat bottom microculture plates. After overnight incubation, target cell lysis was determined according to (14) as described previously (12). In short, reduced incorporation of neutral red by surviving BMM $\varnothing$ was taken as parameter for cytolysis. M. tuberculosis and $M$. leprae reactive CD8 T-cell clones lysed $\mathrm{BMM} \varnothing$ expressing the appropriate antigen. In contrast, L. monocytogenes primed or unprimed BMM $\varnothing$ were not lysed by these 

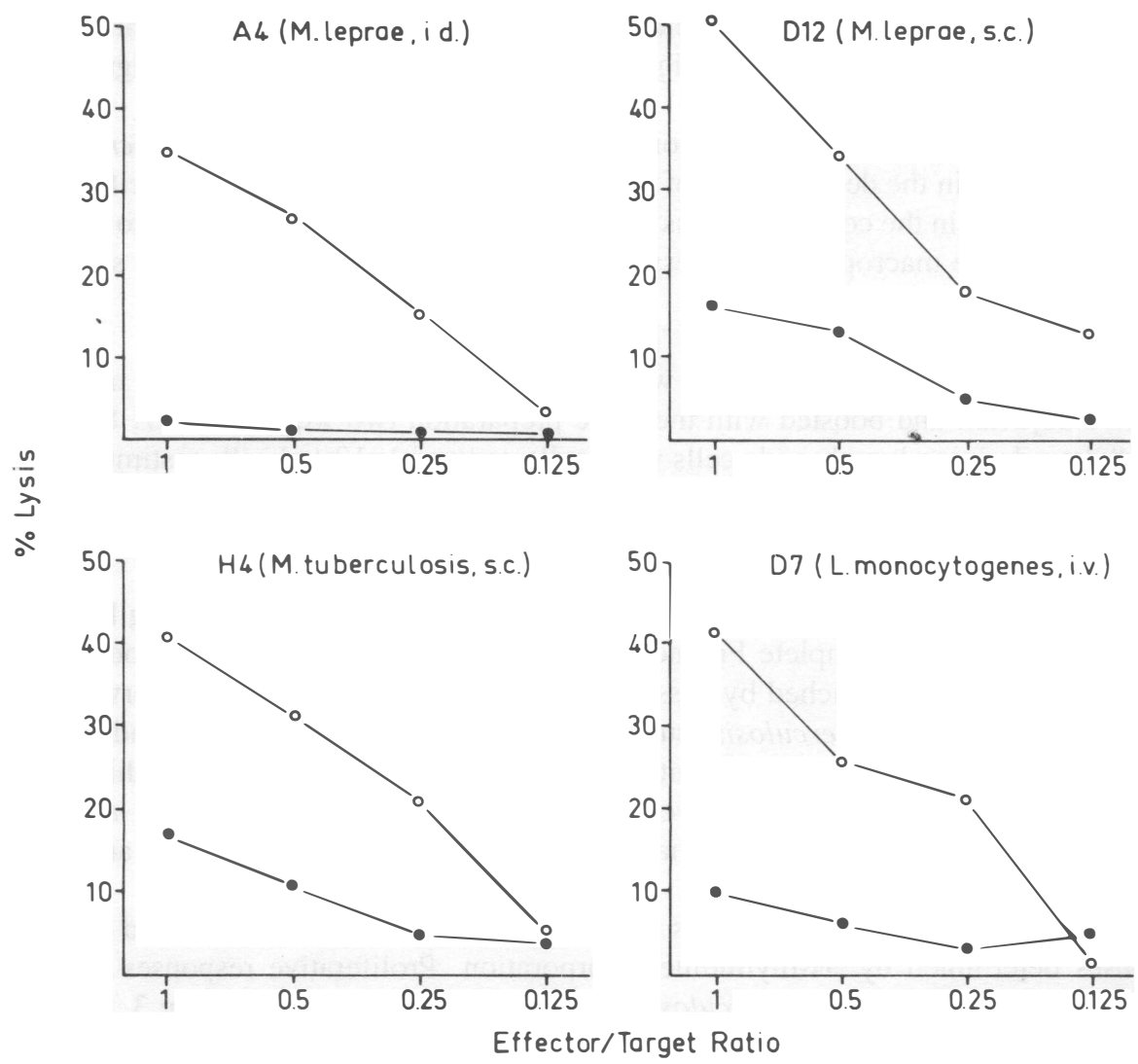

Figure 1: Cytolytic activity of cloned CD8 T cells from M. leprae and M. tuberculosis immune mice. Graded numbers of clones A4 or D12 (M. leprae reactive), clone H-4 (M. tuberculosis reactive) or clone $D 7$ ( $\mathrm{L}$. monocytogenes reactive) were cultured with $B M M \emptyset$ in the presence (O) or absence (•) of soluble $\mathrm{M}$. leprae proteins (clones A4 and D12), killed M. tuberculosis (clone H4), or killed L. monocytogenes (clone D7) as a source of antigen overnight. Afterwards, specific cytolysis was determined (10, 11).

T cells whereas L. monocytogenes primed BMM $\varnothing$ were lysed by L. monocytogenes specific $\mathrm{T}$ cells. The cytolytic activities of representative $\mathrm{T}$-cell clones are documented in Figure 1.

For some T-cell clones from $M$. tuberculosis immune mice we have mapped their restriction to the H-2D locus (11). These data suggest that mycobacteria reactive CD8 T cells are capable of specifically lysing host cells expressing mycobacterial antigen in association with class I molecules.

We assessed whether cytolytic CD8 T-cell clones from M. tuberculosis and M. leprae immune mice can secret IFN- $\gamma$. Graded numbers of cloned T cells were stimulated with antigen, AC, and r-IL-2 and after $24 \mathrm{~h}$ supernatants were collected. IFN-activities were determined by their capacity to inhibit the cytopathic effect of vesicular stomatitis virus on L929 cells as described (13). We found that only few CD8 T-cell clones produced IFN after stimulation with antigen plus $\mathrm{AC}$ alone, After costimulation with antigen, AC, and r-IL-2, how- 
ever, the vast majority of CD8 T-cell clones produced IFN. IFN-secretion by three representative T-cell clones is documented in Figure 2. It can also be concluded from Figure 2 that we are dealing with IFN- $\gamma$.

In conclusion, these findings demonstrate that immunization with $M$. tuberculosis and $M$. leprae results in the development of T lymphocytes of CD8 phenotype specific for mycobacterial antigens in the context of class I molecules. After restimulation in vitro these $\mathrm{T}$ cells are able to (i) lyse macrophages expressing mycobacterial antigens and (ii) to secret IFN- $\gamma$.

\section{MYCOBACTERIA REACTIVE CD4 T-CELL CLONES}

Mice were immunized subcutaneously with $10^{7}$ irradiated $M$. leprae organisms in incomplete Freund's adjuvant and boosted with the same preparation two weeks later $(7,8)$. After another 8 days draining lymph-node cells were collected and $1 \times 10^{6} / \mathrm{ml}$ cells restimulated in vitro with $10^{6} / \mathrm{ml} M$. leprae organisms and $10 \%$ semipurified ConA-SN. Cells were cloned and recloned under limiting dilution conditions in the presence of $\mathrm{AC}$, antigen, and semipurified ConA-SN and cloned cells expanded as described above.

In another approach (9), mice were immunized subcutaneously with $1 \mathrm{mg}$ killed $M$. $t u$ berculosis $\mathrm{H} 37 \mathrm{Ra}$ in incomplete Freund's adjuvant and after 8 days lymph node cells were collected. T cells were enriched by passage over nylon wool columns and afterwards, $5 \times 10^{5}$ AC and $5 \mu \mathrm{g}$ killed $M$. tuberculosis, cultured for 4 days. Cultures were expanded with AC, antigen, and $10 \%$ ConA-SN. Different cell lines were cloned under limiting dilution conditions and expanded as described above. T-cell clones from $M$. lepraeand $M$. tuberculosis immune mice established in this way had the phenotype Thyl ${ }^{+}, \mathrm{L}^{2} \mathrm{~T}_{4}^{+}, \mathrm{Lyt}^{-}$and hence belonged to the CD4 T-cell set.

Graded numbers of cloned $\mathrm{T}$ cells were stimulated with $\mathrm{AC}$, antigen and proliferative responses determined by ${ }^{3} \mathrm{H}$-thymidine incorporation. Proliferative responses of cloned $\mathrm{T}$ cells from $M$. leprae and $M$. tuberculosis immune mice are shown in Figure 3. Proliferative responses were only demonstrable when $\mathrm{T}$ cells and $\mathrm{AC}$ shared the $\mathrm{H}-21-\mathrm{A}$ locus of the MHC indicating that these $\mathrm{T}$ cells were class II restricted.

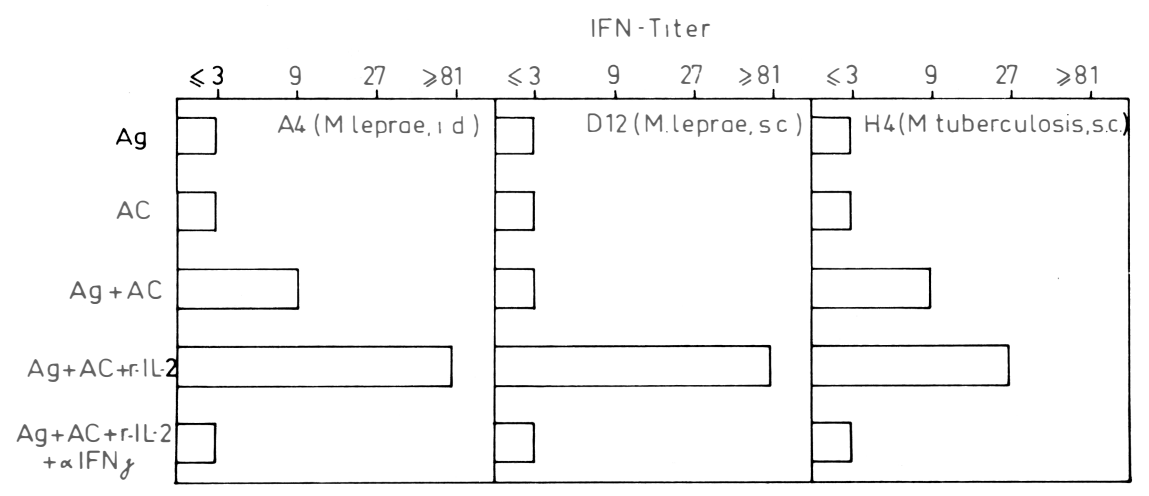

Figure 2: IFN-secretion by cloned CD8 T cells from $\mathrm{M}$. leprae and $\mathrm{M}$. tuberculosis immune mice. Clones $A 4$ or DI2 (M. leprae reactive) or clone $H 4$ (M. tuberculosis reactive) were cultured with AC and soluble M. leprae antigen (clones A4 and D12) or killed M. tuberculosis (clone H4) in the presence or absence of $r-I L-2$. After $24 \mathrm{~h}$, supernatants were collected and IFN activities determined $(10,11)$. In addition, the IFN-test was performed in the presence of anti-IFN- $\gamma$. 


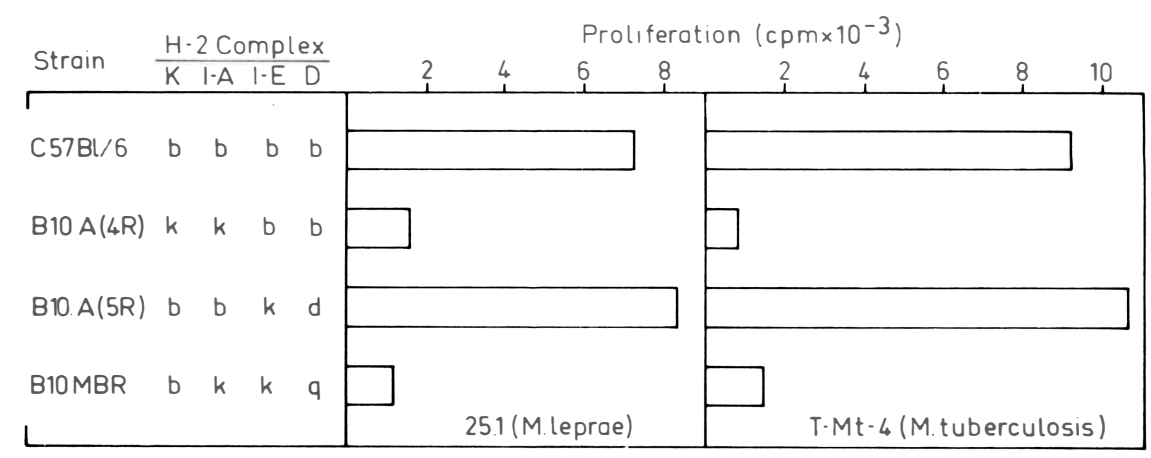

Figure 3: $\mathrm{H}-2$ restricted proliferation by cloned CD4 T cells from $\mathrm{M}$. leprae and $\mathrm{M}$. tuberculosis immune mice. Clone 25.1 (M. leprae reactive) or clone T-Mt-4 (M. tuberculosis reactive) were cultured with $A C$ of different $H$-2 haplotype in the presence of killed $\mathrm{M}$. leprae or killed M. tuberculosis, respectively, as a source of antigen and proliferation was determined (7).

Cloned T cells were stimulated with AC plus antigen and after $24 \mathrm{~h}$ supernatants collected. Supernatants from cultures of T cells, antigen and AC contained significant IL-2 and IFN activities whereas in the absence of either antigen, AC, or T cells no such activities were demonstrable (Figure 4). In the case of $\mathrm{T}$ cell clones from $M$. tuberculosis immune mice we showed that the IFN belonged to the IFN- $\gamma$ class (10). Thus, CD4 T cells could secret the lymphokines IL-2 and IFN- $\gamma$, and IFN- $\gamma$ secretion was independent of exogenous IL- 2 .

We have asked the question whether mycobacteria reactive T-cell clones of CD4 phenotype can kill macrophages expressing mycobacterial antigens. Indeed, BMM $\varnothing$ pulsed with mycobacterial antigens were lysed by cloned CD4 $\mathrm{T}$ cells from $M$. tuberculosis immune mice. Lysis, however, was only observed when BMM $\varnothing$ had been preactivated with IFN- $\gamma$. Phenotype analysis of these BMM $\varnothing$ with the fluorescence activated cell sorter revealed that unstimulated BMM $\varnothing$ were totally devoid of Ia antigens, and that IFN- $\gamma$ markedly stimulated Ia expression on these BMM $\varnothing$. In Figure 5, cytolysis of IFN- $\gamma$ stimulated, antigen pulsed $\mathrm{BMM} \varnothing$ by the CD4 T-cell clone T-Mt-4 is shown. We conclude that mycobacteria specific T cells can lyse antigen presenting host cells provided that these cell express class II molecules.

\section{INDUCTION OF MYCOBACTERIAL GROWTH INHIBITION BY LYMPHOKINES OR BY CD8 T CELLS}

Although it is generally assumed that lymphokine activated macrophages acquire their capacity to degrade intracellular pathogens, attempts to demonstrate mycobacterial killing by activated macrophages have been disappointing (15). Because we found that BMM $\varnothing$ are in a resting state and that they are responsive to IFN- $\gamma$ (see above) we have used these cells to approach the question of activation of anti-mycobacterial capacities in macrophages by lymphokines. For these studies, BMM $\varnothing$ were generated in the absence of serum and antibiotics. BMM $\varnothing$ were activated by lymphokines from mycobacteria-reactive T-cell clones or by $r$-IFN- $\gamma$ f or $24 \mathrm{~h}$. Afterwards, BMM $\varnothing$ were infected with live $M$. bovis organisms. After 4 or 5 days, survival of $M$. bovis organisms was determined. Lymphokines from antigen-stimulated T cells as well as r-IFN- $\gamma$ rendered BMM $\varnothing$ capable of inhibiting growth of $M$. bovis. Figure 6 summarizes data obtained in a typical experiment. These as well as further ex- 
25.1 ( $M$. leproe, s.c.)
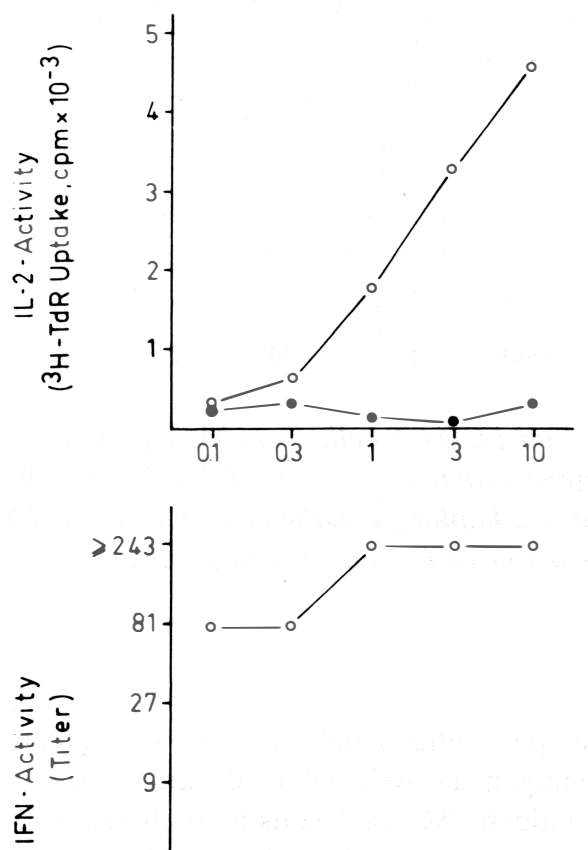

T.Mt- 4 (M.tuberculosis, s.c.)
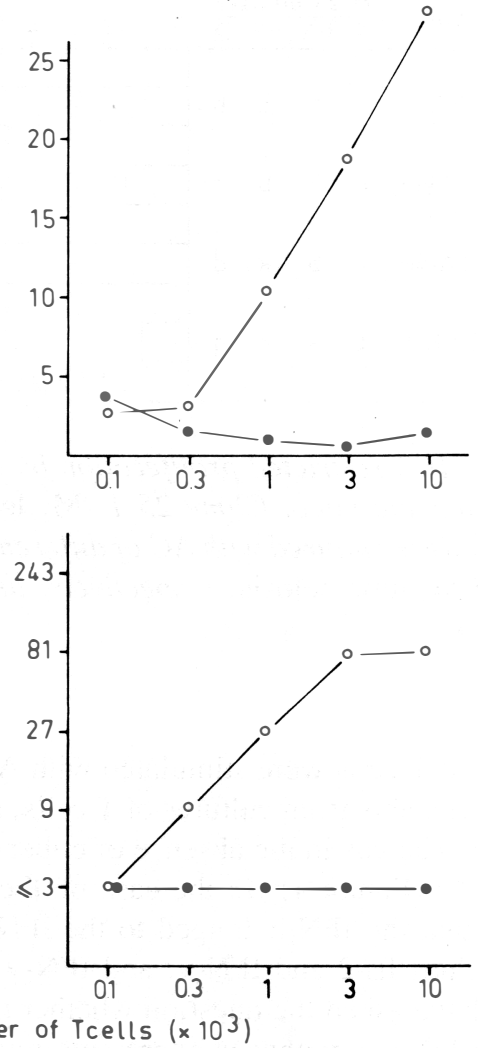

Figure 4: IL-2 and IFN-secretion by cloned CD4 T cells from $\mathrm{M}$. leprae and $\mathrm{M}$. tuberculosis immune mice. Graded numbers of clone 25.1 (M. leprae reactive) or clone T-Mt-4 (M. tuberculosis reactive) were cultured with $A C$ and killed $\mathrm{M}$. leprae or killed $\mathrm{M}$. tuberculosis, respectively (O). After $24 \mathrm{~h}$, supernatants were collected and IL-2 and IFN activities determined $(7,8)$. (•) Supernatants from unslimulated T-cell cultures.

periments (I. Flesch and S.H.E. Kaufmann, submitted for publication) suggest that T-cell derived IFN- $\gamma$ is capable of inducing tuberculocidal or, at least, tuberculostatic capacities in macrophages.

We wanted to analyze possible direct effects of cytolytic T cells on mycobacterial survival in infected macrophages. BMM $\varnothing$ were cultured in the absence of serum and antibiotics and then they were infected with live $M$. bovis organisms. After another 4 days, graded number of $M$. tuberculosis reactive cytolytic CD8 T cells were added. On the next day, survival of $M$. bovis organisms was determined. As illustrated in Figure 7, in the presence of cytolytic T cells, mycobacterial growth was markedly inhibited. This finding suggests that lysis by T lymphocytes of infected host cells markedly affects growth of intracellular mycobacteria.

\section{Discussion}

The data summarized in this article demonstrate that T cells of both CD4 and CD 8 phenotype are generated after immunization with $M$. leprae and $M$. tuberculosis. It is shown that $M$. 


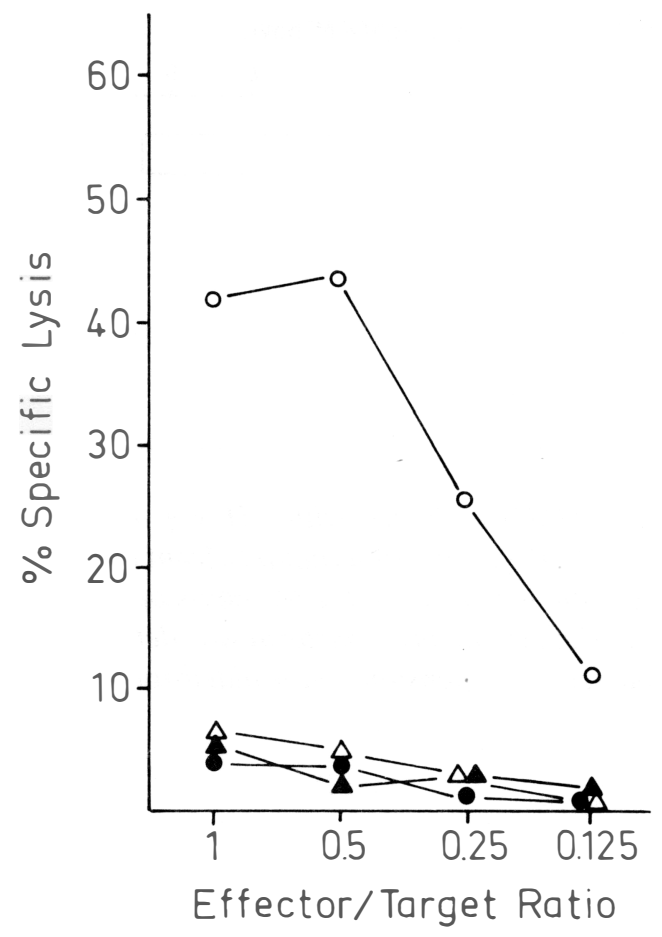

Figure 5: Cytolytic activity by cloned CD4 T cells from $\mathrm{M}$. tuberculosis immune mice. $B M M \emptyset$ were stimulated with IFN- $\gamma$ (open symbols) or remained untreated (closed symbols). Graded numbers of clone T-Mt-4 ( $M$. tuberculosis reactive) were cultured with $B M M \emptyset$ in the presence $(0)$ or absence $(\triangle)$ of killed M. tuberculosis overnight. Afterwards, specific cytolysis was detemined as described in Figure 1.

tuberculosis- and M. leprae-reactive CD4 T cell clones recognize mycobacterial antigens in the context of self class-II molecules and that this recognition triggers the secretion of lymphokines, including IFN- $\gamma$. Our experiments with $r-I F N-\gamma$ and T-cell derived lymphokines reveal that these molecules can indeed render macrophages capable of limiting growth of $M$. bovis and M. tuberculosis H37Rv. M. tuberculosis Middelburg, however, proved resistant against IFN- $\gamma$ activated macrophage functions (I. Flesch and S.H.E. Kaufmann, manuscript submitted). In addition, our study demonstrates that CD8 T cells are capable of lysing macrophages expressig mycobacterial antigens. Cytolytic activity was, however, not unique to CD8 T lymphocytes since CD4 T cells were also able to lyse macrophages primed with mycobacterial antigens provided the latter expressed sufficient amounts of Ia antigens. Furthermore, not only CD4 but also CD8 $\mathrm{T}$ cell clones produced IFN- $\gamma$ after appropriate stimulation. From these studies we conclude that mycobacteria reactive T-cell clones of CD4 and CD8 phenotype are functionally similar and that they primarily differ in their antigen reactivity pattern. This difference may have consequences for the capacity of these lymphocytes to detect mycobacteria-infected host cells: unike CD4 T cells which are restricted to Ia bearing cells CD8 T cells should be able to detect infections in virtually all nucleated host cells.

Our findings obtained with cloned $\mathrm{T}$ cells may shed some light on the relative role of CD4 and CD8 T cells as well as of helper/inducer and cytolytic functions in the immune response against $M$. tuberculosis and $M$. leprae. Based on these findings we shall make four statements which we will discuss separately:

Statement 1: Helper/inducer T-cell functions are protective. Often, M. tuberculosis and $M$. leprae are engulfed by mononuclear phagocytes possessing a high antibacterial potential. These cells can present mycobacterial antigen in association with MHC encoded molecules 


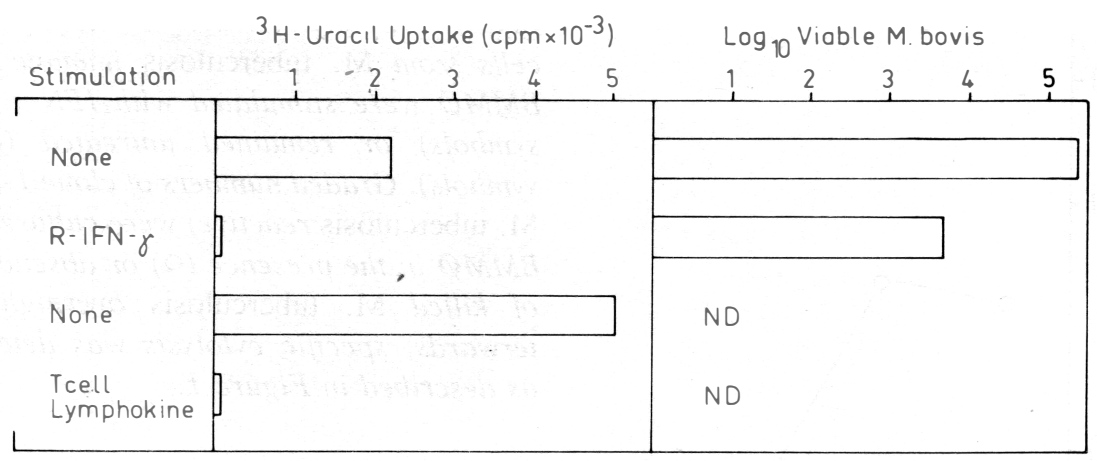

Figure 6: Inhibition of mycobacterial growth by BMMØ activated with $r-I F N-\gamma$ or native lymphokines. BMMØ were activated with $100 \mathrm{U} r-I N F-\gamma$, with supernatants from antigenstimulated T-Mt-4 cells or remained untreated. On the next day, viable M. bovis organisms were added to the cultures. On day 4, the number of surviving mycobacteria was determined by ${ }^{3} \mathrm{H}$-uracil incorporation (16) or by plating bacterial suspensions on Middelbrook $7 \mathrm{HIO}$ agar plates.

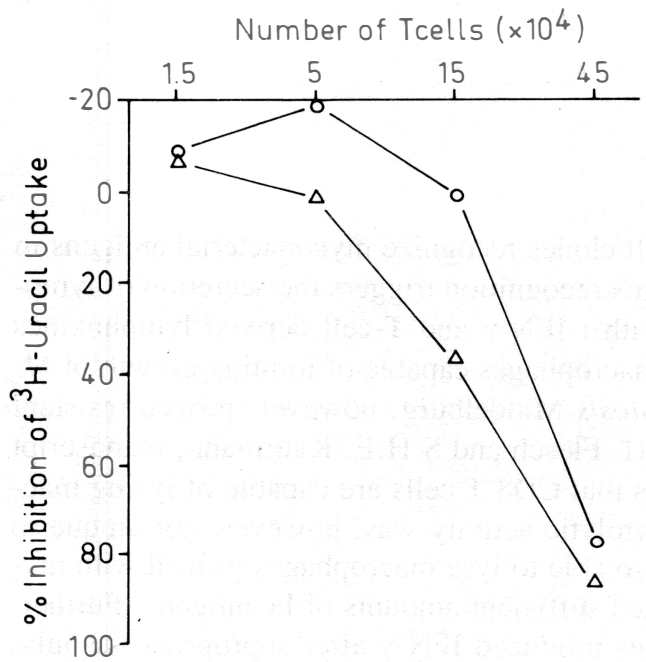

Figure 7: Inhibition of mycobacterial growth in BMMØ by CD8 T cells from $\mathrm{M}$. tuberculosis immune mice. BMMØ were infected with live M. bovis organisms. On d 4, graded numbers of short-term cultured $(\triangle)$ or cloned (o) M. tuberculosis reactive $T$ cells were added. On the next day, surviving mycobacteria were determined by ${ }^{3} \mathrm{H}$-uracil uptake.

of both types. Antigen-specific class II restricted macrophage-T cell interactions trigger the release of multiple lymphokines including IL- 2 and IFN- $\gamma$ by CD4 T cells. Antigen-specific class I restricted macrophage-T-cell interactions result in IFN- $\gamma$ secretion by CD8 T-cells provided IL-2 is supplied as a second signal by CD $4 \mathrm{~T}$ cells. Macrophage activation by lymphokines leads to growth inhibition of intracellular mycobacteria. Although IFN- $\gamma$ seems to play an important role, effects of other, less well defined lymphokines, cannot be excluded. While we assume that this type of reaction is important for host defence against tuberculosis and leprosy it may not be sufficient for complete clearance of highly virulent mycobacteria, particularly if these reside in host cells with low antibacterial potential. 
Statement 2: Cytolytic T-cell functions are protective. Resident tissue macrophages often express only weak anti-microbial functions. Furthermore, mycobacteria sometimes are found in nonmyeloid cells, e.g., M. leprae in Schwann cells. Lysis of infected host cells with weak antibacterial capacities in some cases may be of direct protective value, when the pathogen is unable to multiply in the extracellular space. M. leprae cannot be grown in vitro and it appears that also in the host it is an obligate intracellular pathogen. Therefore, lysis of $M$. leprae infected Schwann cells may be of direct beneficial value for the host. The data summarized in Figure 7 furthermore indicate that cytolysis may directly affect survival of intracellular $M$. tuberculosis organisms. Because cytolytic CD8 T cells are generated during mycobacterial infections host cells of any type and independent from Ia-expression can be detected and attacked by these cells. Ia-expressing cells may be lysed by both CD4 and CD8 $\mathrm{T}$ lymphocytes. At the moment, we do not have sufficient information to definitely decide whether direct killing of mycobacteria through target cell lysis indeed plays a role under in vivo conditions.

Statement 3: T-cell functions are harmful to the host. Although there is no doubt about the importance of $\mathrm{T}$ cells for acquired resistance against tuberculosis and leprosy, several situations can be envisaged where $\mathrm{T}$ cells may exert harmful effects. [A] Uncontrolled lysis by cytolytic $T$ cells of infected macrophages with high antibacterial potential could result in bacterial dissemination from more confined loci like granulomas over the whole body and hence facilitate infection of various organs. [B] Tissue destruction and necrosis can limit important host functions to a significant degree. Both cytolytic T lymphocytes and lymphokine-activated macrophages could participate in destructive processes. Destructive processes will be particularly harmful, whenever highly specialized cells are affected. Thus, lysis of $M$. leprae infected Schwann cells could be responsible for the nerve lesions observed in tuberculoid leprosy. [C] Lysis of antigen presenting cells could impair stimulation of CD4 and CD8 T cells. Thus, cytolytic $\mathrm{T}$ cells may be responsible for certain types of suppression and in this way induce downregulation of the immune response to $M$. tuberculosis or $M$. leprae. Suppressor $\mathrm{T}$ cells have been implicated in the development of lepromatous leprosy $(6,17)$.

Statement 4: Protection depends on both helper/inducer and cytolytic T-cell functions. Lysis of infected tissue macrophages leads to release of bacteria which can subsequently be taken up by immigrant blood monocytes. Provided that the former cells have a low antibacterial potential and the latter ones have a high antibacterial potential, this mechanism would allow bacterial transmission from a rather protective to a more hostile environment. Activation of blood monocytes by lymphokines can then trigger intracellular bacterial degradation. This rather complex sequel of events which depends on different $\mathrm{T}$ cells and macrophages appears to play a major role in acquired resistance against tuberculosis and leprosy.

We believe that all four statements are relevant to tuberculosis and leprosy. Under in-vivo conditions the principle reactions described will not occur independently from each other and must be highly regulated. The final outcome of disease will greatly depend on an intricate balance between the cell types involved in these reactions. We hope that the availability of T-cell clones of CD4 and CD8 type from mice (7-11) and/or man $(18,19)$ will facilitate a deeper understanding of the underlying mechanisms and help us to develop means for the eradication of these diseases.

\section{Acknowledgements}

G. De Libero is a recipient of an EMBO long term fellowship. The expert technical assistance of U. Väth and E. Hug is gratefully acknowledged. We thank Dr. J. Langhorne for critically reading the manuscript, $U$. Brugger for his help with the fluorescence activated cell sorter, and R. Brugger and R. Schneider for typing the manuscript. R-IL-2 was a kind gift from 
Sandoz AG and from Hoffmann-La Roche. Rabbit anti-IFN- $\gamma$ antiserum and $r$-IFN- $\gamma$ were produced by Genentech Inc. and kindly provided by Boehringer Ingelheim. We thank Drs. F.W. Fitch, P.D. Gottlieb, P. Marrack, and J. Kappler for hybridoma cells. Irradiated $M$. leprae organisms and soluble $M$. leprae antigens were kindly provided by Dr. R.J.W. Rees, Harrow, U.K., through the WHO-IMMLEP program; killed lyophilized M. leprae organisms were a kind gift of Dr. P.J. Brennan, Fort Collins, CO. This work was supported by the Immunology of Leprosy component of the UNDP/World Bank/WHO Special Program for Research and Training in Tropical Diseases and by the WHO as part of its Program for Vaccine Development.

\section{References}

1 Fitch FW. T cell clones and T-cell receptors. Microbiol Rev, 1986, 50, 50.

2 Zinkernagel RM, Doherty PC. MHC restricted cytotoxic T cells: Studies on the biological role of polymorphic major transplantation antigens determining T-cell restriction-specificity, function and responsiveness. Adv Immunol, 1979, 27, 50.

3 Hahn H, Kaufmann SHE. Role of cell mediated immunity in the defence against infections. Rev Infect Dis, 1981, 3, 1221.

4 Moulder JW. Comparative biology of intracellular parasitism. Microbiol Rev, 1985, 49, 298.

5 Lepay DA, Nathan CF, Murray HW, Steinman RM, Cohn ZA. Murine Kupffer Cells: mononuclear phagocytes deficient in the generation of reactive oxygen intermediates. $J$ Exp Med, 1985, 161, 1079.

6 Kaufmann SHE. Immunology of leprosy: new facts, future perspectives. Microbial Pathogenesis, 1986, 1, 107.

7 Kaufmann SHE. Biological activities of a murine T cell clone with reactivity to Mycobacterium leprae. Cell Immunol, 1984, 83, 215.

8 Kaufmann SHE, Brinkmann V, Kiderlen AF, Lohmann-Matthes ML, Müller I, Wrazel LJ. Role of T lymphocyte clones in cell-mediated immunity. In: Mononuclear phagocytes and inflammation (Ed. R v Furth), Martin Nijhoff Publishers, Dordrecht, Boston, Lancaster, 1985, p 541.

9 Kaufmann SHE, Flesch I. Function and antigen recognition pattern of L3T4+ T-cell clones from Mycobacterium tuberculosis immune mice. Infect Immun, 1986, 54, 291.

10 Chiplunkar S, De Libero G, Kaufmann SHE. Mycobacterium leprae-specific Lyt2+ T lymphocytes with cytolytic activity. Infect Immun, in press.

11 Kaufmann SHE, De Libero G. Cytolytic T cells in Mycobacterium tuberculosis infections. In: Tuberculosis: Interactions with the Immune System (Eds. H Friedman and M Bendinelli) Plenum, New York, in press.

12 De Libero G, Kaufmann SHE. Antigen-specific Lyt2+ cytolytic T lymphocytes from mice infected with the intracellular bacterium Listeria monocytogenes. J Immunol, 1986, $137,2688$.

13 Kaufmann SHE, Hug E, De Libero G. Listeria monocytogenes reactive T lymphocyte clones with cytolytic activity against infected target cells. J Exp Med, 1986, 164, 363.

14 Parish CR, Müllbacher A. Automated colorimetric assay for T cell cytotoxicity. J Immunol Meth, 1983, 58, 225.

15 Douvas GS, Looker DL, Vatter AE, Crowle AJ. Gamma interferon activates human macrophages to become tumoricidal and leishmanicidal but enhances replication of macrophage associated mycobacteria. Infect Immun 1985, 50, 1.

16 Rook GAW, Rainbow S. An isotope incorporation assay for the antimycobacterial effects in human monocytes. Ann Immunol (Paris), 1981, 132 D, 281. 
17 Bloom BR, Mehra V. Immunological unresponsiveness in leprosy. Immunol Rev, 1984, $84,5$.

18 Emmrich F, Kaufmann SHE. Human T cell clones with reactivity to Mycobacterium leprae as tools for the characterization of candidate vaccines against leprosy, Infect Immun, 1986, 51, 879.

19 Emmrich F, Thole J, Embden Jv, Kaufmann SHE. A recombinant 64 kD protein of Mycobacterium bovis BCG specifically stimulates human $\mathrm{T} 4$ clones reactive to mycobacterial antigens. J Exp Med, 1986, 163, 1024. 